\title{
Characteristics of human umbilical cord mesenchymal stem cells during $e x$ vivo expansion
}

\author{
SHENGYING LI ${ }^{1 *}$, YUXIA WANG ${ }^{2 *}$, LIPING GUAN ${ }^{3}$ and MINGLI JI ${ }^{4}$ \\ Departments of ${ }^{1}$ Pharmacology, ${ }^{2}$ Pathophysiology, ${ }^{3}$ Functional Science Laboratory and ${ }^{4}$ Physiology, \\ Xinxiang Medical University, Xinxiang, Henan 453003, P.R. China
}

Received May 15, 2014; Accepted January 30, 2015

DOI: $10.3892 / \mathrm{mmr} .2015 .3999$

\begin{abstract}
Human umbilical cord mesenchymal stem cells (hUCMSCs) have potential clinical applications in different types of diseases. In order to acquire enough cells, hUCMSCs have to be expanded ex vivo. However, it remains to be elucidated whether the characteristics of hUCMSCs are altered during ex vivo expansion. In the present study, the quality of hUCMSCs, which is important for successful therapeutic use, was systematically examined during hUCMSC expansion ex vivo. Morphologically, hUCMSCs exhibited no visible changes during culture. In addition, hUCMSCs retained their proliferative ability between passages $0-5$. At the molecular level, the cells continued expressing the specific positive surface markers, CD29, CD73 and CD90, and did not express the negative surface markers, CD14, CD34 or CD45, during culture ex vivo. Furthermore, the hUCMSCs exhibited low immunogenicity, which was maintained when cultured for five passages. However, the immunological properties of hUCMSCs were altered at passage 10, at which the percentage of hUCMSCs expressing human leukocyte antigen-I was significantly increased. Collectively, these results suggested that hUCMSCs used for cell-based therapies require obtaining from cells, which have been expanded for fewer than five passages.
\end{abstract}

\section{Introduction}

Mesenchymal stem cells (MSCs) are multipotent stromal cells (1). MSCs can differentiate into different types of cells (1), including osteoblasts (2), chondrocytes, adipocytes (3) and neuronal cells $(4,5)$. Morphologically, MSCs are thin and long

Correspondence to: Professor Mingli Ji, Department of Physiology, Xinxiang Medical University, 601 Jinsui Road, Xinxiang, Henan 453003, P.R. China

E-mail: teachermingliji@163.com

*Contributed equally

Key words: human umbilical cord, mesenchymal stem cells, immunogenicity, transplantation, cell therapy with small cell bodies (1). At the molecular level, MSCs express specific surface markers, including CD29, CD73 and CD90, however, they do not express CD14, CD34 or CD45 (6-11). In addition, MSCs have immunological characteristics of immune suppression and immune avoidance $(12,13)$.

MSCs are widely used for the treatment of numerous diseases, including tissue injury and immune disorders (14). For example, the differentiation of MSCs to connective tissues has been applied in tissue engineering; MSCs are implanted in to a tissue-specific scaffold and regenerate damaged cartilage and bones $(15,16)$. In addition, MSCs secrete various factors, which assist in repairing or regenerating cardiac tissues, brain tissues and menisci (17-19). Ex vivo and preclinical studies have demonstrated that MSCs are important in the proliferation of immune cells and act as immunomodulators in tissue transplantation (20-22). Although MSCs are a promising source for cell-based therapy of diseases, potential challenges remain, including obtaining sufficient qualified MSCs with strong proliferation potential, low immunogenicity and significant MSCs characteristics for clinical application (23).

MSCs can be generated from bone marrow, which is a major source of MSCs (24). However, the extraction of cells from bone marrow is associated with painful harvesting and ethical constraint (25). In addition, MSCs generated from bone marrow in elderly patients are fewer in number and have poor differentiation potential (26). Therefore, it is difficult to obtain a sufficient quantity of qualified cells for successful therapies. Human umbilical cords (hUCs) are considered as another ideal source for the derivation of MSCs (27). There are several advantages for hUCs as a source to generate MSCs: There is little ethical concern regarding the use of hUCs in research and in clinical therapies, as hUCs are considered as medical waste (25); human umbilical cord MSCs (hUCMSCs) proliferate rapidly during ex vivo culture and are considered to be immune privileged (28-30); and hUCMSCs may be cryogenically stored in cell banks, thawed and expanded for therapeutic use (25). These characteristics make hUCMSCs an attractive candidate for the clinical application and therapeutic use of MSCs. However, to ensure the safe and successful therapeutic use of hUCMSCs, several concerns require addressing. It is understood that hUCMSCs require expansion ex vivo in order to obtain enough cells for application; however, few studies have investigated whether the characteristics of hUCMSCs are altered during ex vivo culture. In addition, the optimized 
time point for the use of hUCMSCs, in order to balance the quantity and quality of the expanded hUCMSCs, remains to be elucidated.

In order to elucidate these unknown factors, the present study examined four characteristics (morphology, proliferative ability, surface markers and immunological properties) of hUCMSCs during long-term ex vivo culture. hUCMSCs maintained their characteristics for a minimum of five passages during ex vivo culture. The present study assists in understanding the characteristics of hUCMSCs during long-term ex vivo culture and provides a foundation for the clinical application of hUCMSCs.

\section{Materials and methods}

Materials and reagents. Dulbecco's modified Eagle's medium (DMEM; cat. no. 12800-017; Gibco-BRL, Carlsbad, CA, USA), fetal bovine serum (FBS; cat. no. HB0205; Sijiqing Biotechnology, Ltd, Hangzhou, China), non-essential amino acids (cat no. 11140-070; Gibco-BRL) and stem cell growth factor (cat no. PHC2113; Gibco-BRL) were used for cell culture. The antibodies used for flow cytometry were as follows: Fluorescein isothiocyanate (FITC) monoclonal mouse anti-human CD14 (1:500; cat. no. IM0645U), monoclonal mouse anti-human CD29 (1:500; cat. no. IM0791U), monoclonal mouse anti-human CD34 (1:500; cat. no. IM1870), monoclonal mouse anti-human CD45 (1:500; cat. no. A07782), monoclonal mouse anti-human CD90 (1:500; cat. no. IM1839U) and FITC mouse anti-human human leukocyte antigen (HLA)-DR (1:500; cat. no. IM1638U) antibodies, purchased from Beckman Coulter, Inc. (Brea, CA, USA), and phycoerythrin (PE) monoclonal mouse anti-human CD73 (1:500; cat. no. 344004) and PE monoclonal mouse anti-human HLA-I (1:500; cat. no. 311406), purchased from BioLegend, Inc. (San Diego, CA, USA).

Isolation and culture of hUCMSCs. The use of hUCs in the present study was approved by the ethics committee of the Third Affiliated Hospital of Xinxiang Medical College (Xinxiang, China). Informed consent was obtained from the mothers prior to labor and delivery of infants and the study was performed according to the principles laid out in the ethical principles of the Declaration of Helsinki. A total of six human umbilical cords were collected from full-term infants, delivered by caesarean section under sterile conditions, to generate different hUCMSCs. The hUC membrane and blood vessels were removed in order to obtain the Wharton's jelly. Subsequently, the Wharton's jelly was sectioned into pieces $\sim 1 \mathrm{~mm}^{3}$. These small pieces were cultured in DMEM/F12 (Invitrogen Life Technologies, Grand Island, NY, US) supplemented with $10 \%$ FBS, $1 \%$ non-essential amino acids and $1 \mu \mathrm{g} / \mathrm{ml}$ stem cell growth factor. The cells were cultured in an incubator at $37^{\circ} \mathrm{C}$ with saturated humidity and $5 \%(\mathrm{v} / \mathrm{v}) \mathrm{CO}_{2}$.

Proliferation analysis of hUCMSCs. Cells between passages 0 and 5 were selected for proliferative analysis. The cells were passaged at $80 \%$ confluence. For passage, the cells were treated with $2.5 \mathrm{~g} / 1$ trypsin (Sigma-Aldrich, St. Louis, MO, USA) at $37^{\circ} \mathrm{C}$ for $10 \mathrm{~min}$, and then centrifuged at $800 \mathrm{x} \mathrm{g}$ for $10 \mathrm{~min}$. The cells were then resuspended in $6 \mathrm{ml}$ fresh culture medium. Following counting of the cells, using a Hausser Scientific Hemocytometer (Thermo Fisher Scientific, Inc, Waltham, MA, USA) according to the manufacturer's instructions, $3 \times 10^{5}$ cells were seeded into $10 \mathrm{ml}$ medium in a $75-\mathrm{cm}$ flask (cat. no. 430641; Corning Life Sciences, Inc., Tewksbury, MA, USA) and cultured in an incubator at $37^{\circ} \mathrm{C}$ with saturated humidity and $5 \%(\mathrm{v} / \mathrm{v}) \mathrm{CO}_{2}$. The cells were incubated and cultured for two days between each passage. The number of cells in each passage was recorded to generate a growth curve using GraphPad Prism 5 software (GraphPad, Inc., La Jolla, CA, USA) and each experiment was performed in triplicate.

Morphology of hUCMSCs. The hUCMSCs were dissociated from the tissue and cultured for 7 days. The morphology of the cells was observed using a microscope (magnification, x100; Olympus BX53, Olympus Corporation, Tokyo, Japan) at P0, P5 and P10. Representative images are presented in Fig. 1.

Detection of the surface markers of hUCMSCs. The MSCs $\left(1 \times 10^{6}\right)$ at P0, P5 or P10 were incubated with FITC/PE-conjugated mouse anti-human antibodies for CD14, CD34, CD45, CD29, CD73, and CD90 for $15 \mathrm{~min}$ at room temperature. Subsequently, the cells were washed twice with phosphate-buffered saline (PBS; Invitrogen Life Technologies), centrifuged at $800 \mathrm{x}$ g for $10 \mathrm{~min}$ and resuspended in $0.5 \mathrm{ml}$ PBS. The antibody-bound cells were then analyzed using a flow cytometer (FC500; Beckman Coulter, Inc.). The data from flow cytometry were analyzed using FlowJo software version 10 (http://www.flowjo.com). The values are presented as the mean \pm standard deviation (SD; $\mathrm{n}=3$ ). Cells incubated with PBS served as a control and all experiments were performed in triplicate.

Analysis of immunological properties. FITC-conjugated HLA-DR and PE-conjugated HLA-I mouse anti-human antibodies were incubated with $1 \times 10^{6}$ MSCs at P0, P5 or P10 for $15 \mathrm{~min}$ at room temperature. Flow cytometry was performed following the cells being washed twice with PBS, The cells were then centrifuged at $800 \mathrm{x} g$ for $10 \mathrm{~min}$ and resuspended in $0.5 \mathrm{ml}$ PBS. Cells incubated with PBS instead of antibodies served as a control. All experiments were performed in triplicate.

Statistical analysis. Data are presented as the mean \pm SD. The results were analyzed using SPSS 13.0 software (SPSS, Inc., Chicago, IL, USA) and statistical significance was assessed using a paired Student's t-test. $\mathrm{P}<0.05$ was considered to indicate a statistically significant difference.

\section{Results}

Morphology of the hUCMSCs isolated from hUC tissue does not change during ex vivo culture. In order to examine the morphology of hUCMSCs isolated from human umbilical cord tissue during long-term ex vivo culture, cells at P0, P5 and P10 were observed using optical microscopy. At P0, the cells started to migrate from the explants following culture of tissue for 6-7 days (Fig. 1A). These cells then became long and thin with small cell bodies, typical morphological characteristics of MSCs, in the following days. Following culture for 14 days, the 
Table I. Percentage of human umbilical cord mesenchymal stem cells expressing different markers at P0, P5 and P10.

\begin{tabular}{|c|c|c|c|c|c|c|c|c|}
\hline \multirow[b]{2}{*}{$P$} & \multicolumn{3}{|c|}{ Positive surface markers (\%) } & \multicolumn{3}{|c|}{ Negative surface markers (\%) } & \multicolumn{2}{|c|}{ Immunological markers (\%) } \\
\hline & CD29 & CD73 & CD90 & CD14 & CD34 & CD45 & HLA-DR & HLA-I \\
\hline P0 & $99.33 \pm 0.80$ & $99.86 \pm 0.18$ & $99.66 \pm 0.40$ & $0.70 \pm 0.92$ & $0.10 \pm 0.08$ & $0.93 \pm 0.32$ & $0.13 \pm 0.12$ & $0.63 \pm 0.36$ \\
\hline P5 & $99.90 \pm 0.14$ & $99.16 \pm 0.49$ & $99.76 \pm 0.26$ & $0.86 \pm 0.49$ & $0.06 \pm 0.04$ & $0.83 \pm 0.53$ & $0.40 \pm 0.43$ & $1.13 \pm 0.49$ \\
\hline P10 & $98.93 \pm 0.44$ & $99.73 \pm 0.12$ & $99.30 \pm 0.49$ & $0.63 \pm 0.69$ & $0.23 \pm 0.12$ & $1.40 \pm 0.35$ & $0.63 \pm 0.44$ & $11.46 \pm 0.92 *$ \\
\hline
\end{tabular}

${ }^{*} \mathrm{P}<0.05$ vs. P0 and P5. Data were analyzed using FlowJo software version 10 and histogram overlays are displayed as a percentage of the maximum of all bins (stands for events or cells). Values are presented as the mean \pm standard deviation ( $n=3$ ). P, passage; HLA, human leukocyte antigen.

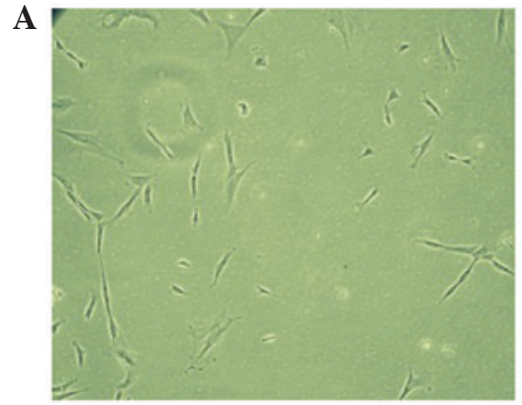

PO

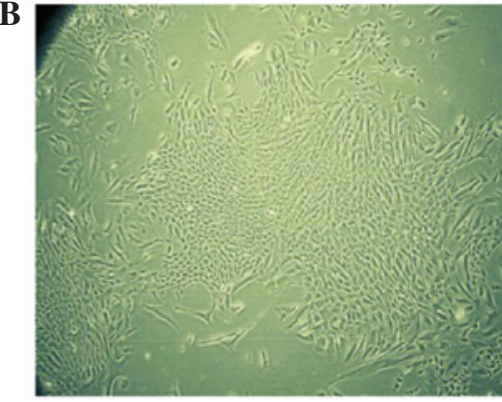

p5

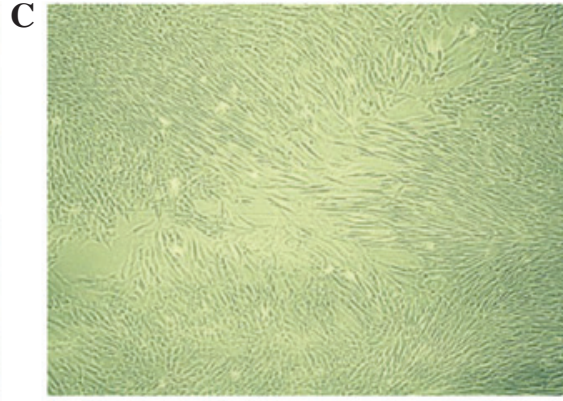

p10

Figure 1. Morphology of hUCMSCs is not altered at different passages. The hUCMSCs were dissociated from the tissue following plating and culture of the Wharton's jelly tissue pieces for 7 days. Images of the cells were captured at (A) P0, (B) P5 and (C) P10 (magnification, x100). The hUCMSCs at P5 appeared spindle-shaped and the cells at P10 retained a similar morphology to that at P5. hUCMSCs, human umbilical cord mesenchymal stem cells; P, passage.

cells reached $80-90 \%$ confluence and were ready for passage. At P5 and P10, the cells exhibited similar morphologies to the P0 cells (Fig. 1B and C). In addition, the cells reached $\sim 80 \%$ confluence after $\sim 14$ days at P0 and after 3-4 days at P5 and $\mathrm{P} 10$. These results suggested cells isolated from hUC tissue exhibit the morphology of MSCs and expand rapidly without visible changes in morphology following long-term ex vivo culture.

hUCMSCs exhibit high proliferative ability during ex vivo culture. In the present study, hUCMSCs were expanded rapidly ex vivo, as the duration required for these cells to reach confluency following passage was shortened between P0 and P5. In order to accurately measure the proliferative activity of these cells, the total cell number was calculated at each passage between $\mathrm{P} 0$ to $\mathrm{P} 5$. The results revealed that the number of cells was $\sim 10^{6}$ at $\mathrm{P} 0$, and increased to $20.6 \pm 0.44 \times 10^{8}$ at $\mathrm{P} 3$, $8.87 \pm 0.59 \times 10^{9}$ at $\mathrm{P} 4$ and $3.61 \pm 0.31 \times 10^{10}$ at $\mathrm{P} 5$. The growth curve demonstrated a clear increase in cell number between P0 and P5 (Fig. 2), indicating the marked proliferative ability of these cells during ex vivo culture. Of note, the quantity of cells at P5 was sufficient for the majority of clinical applications.

Characterization of surface markers of hUCMSCs in ex vivo culture. To determine the properties of hUCMSCs during ex vivo culture, the specific molecular surface markers of hUCMSCs in cultures were examined. Flow cytometric analysis revealed that $99 \%$ of the cells exhibited positive

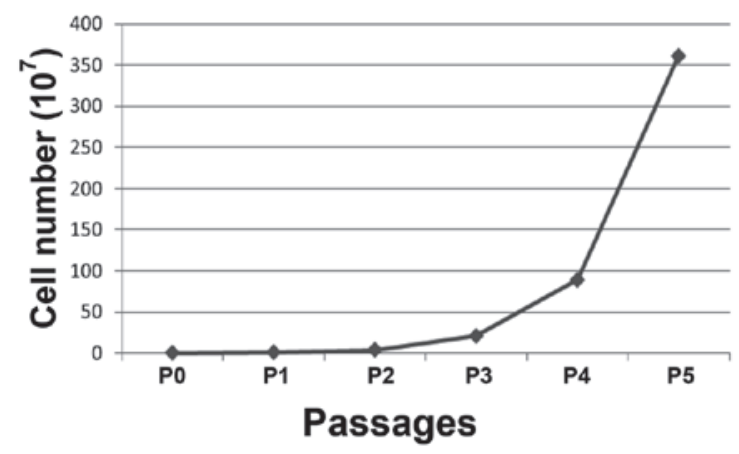

Figure 2. Human umbilical cord mesenchymal stem cells exhibit marked proliferative ability during ex vivo culture. The total number of cells was recorded at each passage between P0 and P5 and a growth curve was constructed. P, passage.

expression of the CD29, CD73 and CD90 hUCMSC markers at P0 (Table I; Fig. 3A-C). Whereas these cells negatively expressed the CD14 monocyte marker, and the CD34 and CD45 endothelial and hematopoietic markers at this stage (Table I; Fig. 3D-F). These results indicated that the isolated cells were purified MSCs. Following expansion ex vivo, the percentages of cells expressing MSC markers at P5 and P10 were maintained at high levels ( $\geq 99 \%$; Table I; Fig. 3A-C). In addition, few cells expressed the monocyte, endothelial and hematopoietic markers at P5 and P10 (Table I; Fig. 3D-F). These results suggested that the molecular characteristics of hUCMSCs were maintained following ex vivo expansion. 
A

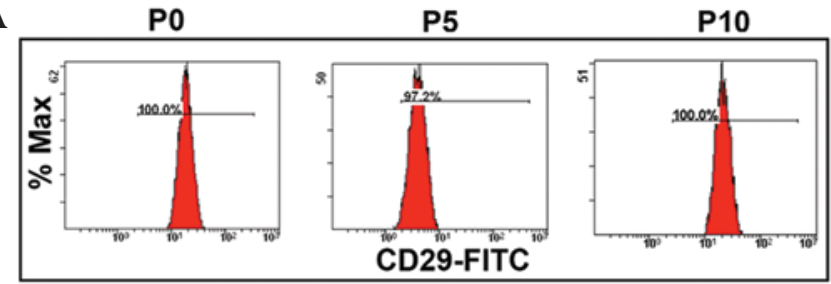

C

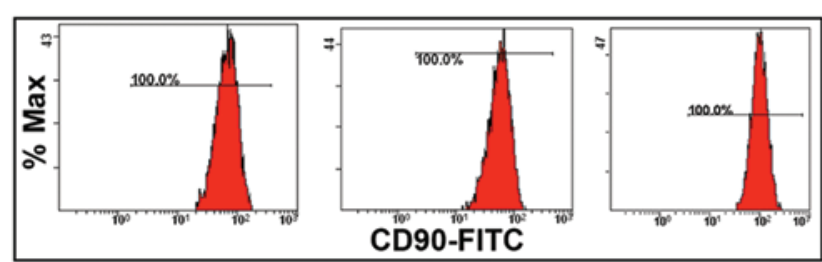

$\mathbf{E}$

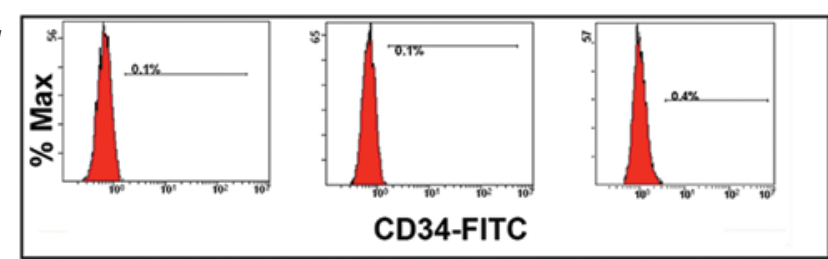

B

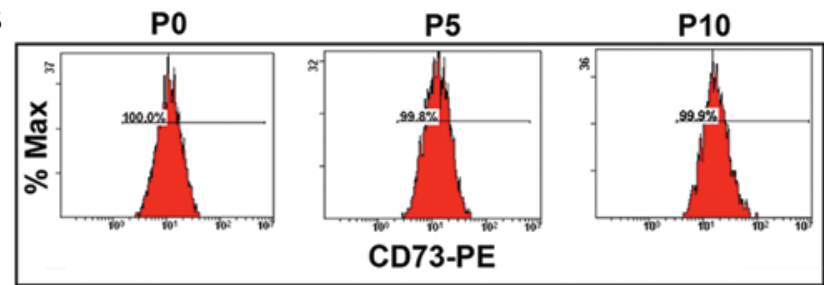

D
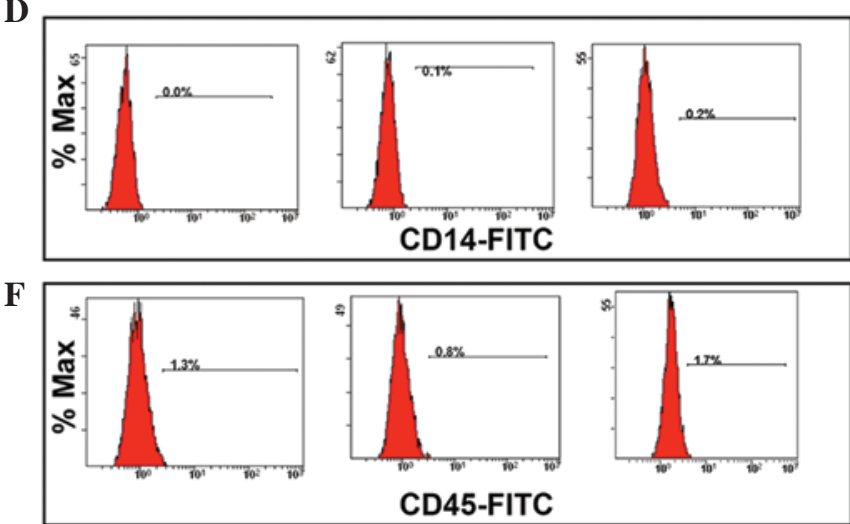

Figure 3. Expression levels of surface markers are maintained during ex vivo culture of hUCMSCs. Flow cytometric analysis of the percentage of cells expressing surface markers in the hUCMSCs at P0, P5 and P10. Data were analyzed using FlowJo software and histogram overlays are displayed as a percentage of the maximum of all bins (stands for events or cells). Representative images of the flow cytometric analysis of (A) CD29, (B) CD73, (C) CD90, (D) CD14, (E) CD34 and (F) CD45 surface markers are shown. hUCMSCs, human umbilical cord mesenchymal stem cells; P, passage; FITC, fluorescein isothiocyanate; PE, phycoerythrin.
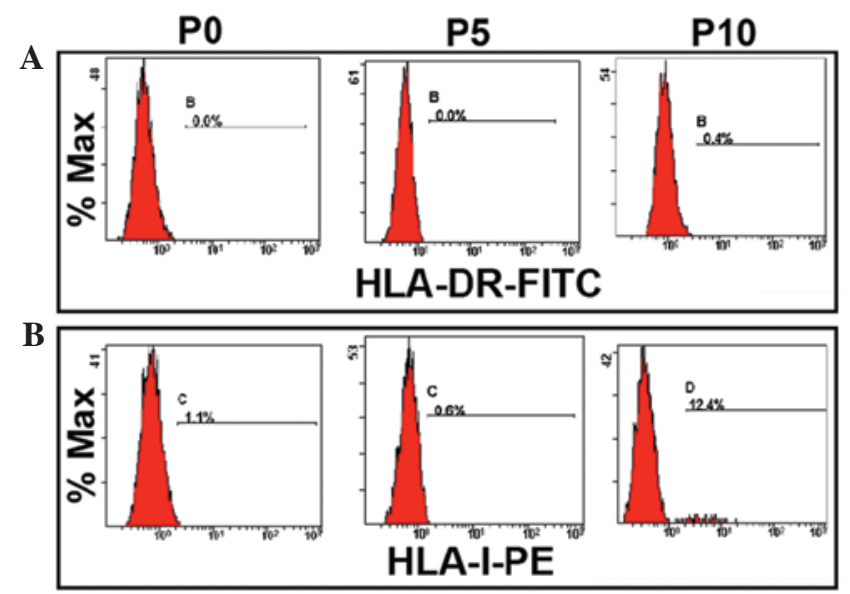

Figure 4. Expression of HLA-I is increased in hUCMSCs at P10. Flow cytometric analysis of the expression of immunological molecules in hUCMSCs at P0, P5 and P10 during ex vivo culture. Data were analyzed using FlowJo software and histogram overlays are displayed as a percentage of the maximum of all bins (stands for events or cells). Representative images of the flow cytometric analysis of (A) HLA-DR and (B) HLA-I are shown. hUCMSCs, human umbilical cord mesenchymal stem cells; P, passage; HLA, human leukocyte antigen; FITC, fluorescein isothiocyanate; PE, phycoerythrin.

Immunological properties of hUCMSCs are altered at P10. The immunological properties of hUCMSCs were examined during ex vivo expansion. The results revealed that the percentage of cells expressing HLA-DR [major histocompatibility complex class I-(MHC I)] was retained at low levels during ex vivo culture at P0, P5 and P10 (Table I; Fig. 4A). In addition, the percentage of hUCMSCs expressing HLA-I (MHC I) at P0 and P5 (Table I; Fig. 4B) were maintained at a low level, suggesting that these cells can be applied for therapy without the requirement of strict MHC I matching.
However, the percentage of hUCMSCs expressing HLA-I was significantly increased to $11.46 \pm 0.92 \%$ at $\mathrm{P} 10$ compared with that of P0 and P5 ( $\mathrm{P}<0.05)$ (Table I; Fig. 4B). This suggested that the immunological properties of hUCMSCs were altered following long-term ex vivo culture.

\section{Discussion}

Previous studies have demonstrated that MSCs are multipotent and may differentiate into a variety of cell types ex vivo, including bone, cartilage, fat and neural cells (1-5). These characteristic of MSCs implies that they have potential applications for tissue engineering and cell-based therapies in the future. Previous studies have demonstrated that MSCs also have therapeutic potential for the treatment of neurodegenerative diseases and cancer (31-34). However, several challenges remain in the clinical application of MSCs, including the acquisition of qualified MSCs. It has been demonstrated that injections of allogeneic UCMSCs elicit an immune response (35), which may be due to the use of unqualified MSCs with altered characteristics (36). One technique to avoid using unqualified MSCs following ex vivo expansion is to monitor their characteristics during ex vivo culture. In the present study, the characteristics of hUCMSCs were examined during long-term ex vivo culture. The morphology of the hUCMSCs remained consistent during culture and exhibited a typical MSC morphology. In addition, the hUCMSCs retained a marked proliferative ability and expanded rapidly ex vivo between P0 and P5. Furthermore, the expression of surface markers was examined in the hUCMSCs cultured ex vivo; the results demonstrated that these cells positively expressed CD29, CD73 and CD90, which are specific surface markers of MSCs, whereas they negatively expressed the CD14 monocyte 
marker and the CD34 and CD45 endothelial and hematopoietic markers. These molecular characteristics were maintained following the passage and culture of MSCs for 10 passages ex vivo. The immunological properties of the hUCMSCs were also investigated during ex vivo culture and revealed that it was possible to passage hUCMSCs to P5 without change of immune characteristics; however, the number of cells expressing HLA-I was significantly increased when the hUCMSCs were passaged to P10. These results indicated that the hUCMSs maintained their characteristics for a minimum of five passages during culture ex vivo and suggested that it may be beneficial to use hUCMSCs, which are expanded for fewer than five passages for allogeneic transplant or other cell-based therapies. Although the present study identified no significant changes were detected in hUCMSC morphology, proliferative activity, surface marker expression or immunological properties between P0 and P5, whether other differences, including heterologous cells, exist among different passages remains to be elucidated.

hUCMSs have the potential for rapid expansion ex vivo (37); this is a vital characteristic of MSCs, as rapid proliferation may generate enough cells for tissue engineering and cell-based therapies. The present study observed that MSCs expanded rapidly during ex vivo culture, with an increase in the number of MSCs between $1 \times 10^{6}$ at $\mathrm{P} 0$ and $1 \times 10^{10}$ at $\mathrm{P} 5$. These figures meets the requirements of most therapies using MSCs or their derived cells. Of note, these MSCs retained qualified status at P5, as their molecular characteristics and immunological properties were maintained. Collectively, the results identified an optimized time window, prior to passage 5, to obtain enough qualified MSCs for clinical application. However, the ex vivo data presented in the present study requires confirmation with additional experiments to determine whether the use of MSCs at $\mathrm{P} 0$ or $\mathrm{P} 5$ have different responses in in vivo transplantation and cell therapy.

In conclusion, the results of the present study demonstrated that hUCMSs exhibited a typical MSCs morphology, retained marked proliferative ability, maintained the expression of specific surface markers and exhibited low immunogenicity between P0 and P5 in ex vivo culture. These characteristics highlight MSCs as a promising candidate for clinical application in allogenic transplantation and other cell-based therapies.

\section{Acknowledgements}

The authors would like to thank all the other members of their laboratory for their technical support and helpful discussions. The present study was supported by a grant from The Education Department Natural Sciences Research Plan Project (Henan, China; grant no. 12B310017).

\section{References}

1. Beyer Nardi N and da Silva Meirelles L: Mesenchymal stem cells: isolation, in vitro expansion and characterization. Handb Exp Pharmacol 249-282, 2006.

2. Brighton CT and Hunt RM: Early histological and ultrastructural changes in medullary fracture callus. J Bone Joint Surg Am 73 832-847, 1991.

3. Brighton CT and Hunt RM: Early histologic and ultrastructural changes in microvessels of periosteal callus. J Orthop Trauma 11: 244-253, 1997.
4. Birbrair A, Wang ZM, Messi ML, Enikolopov GN and Delbono O: Nestin-GFP transgene reveals neural precursor cells in adult skeletal muscle. PLoS One 6: e16816, 2011.

5. Jiang Y, Jahagirdar BN, Reinhardt RL, et al: Pluripotency of mesenchymal stem cells derived from adult marrow. Nature 418: 41-49, 2002.

6. Zhou C, Yang B, Tian Y, et al: Immunomodulatory effect of human umbilical cord Wharton's jelly-derived mesenchymal stem cells on lymphocytes. Cell Immunol 272: 33-38, 2011.

7. Liu S, Yuan M, Hou K, et al: Immune characterization of mesenchymal stem cells in human umbilical cord Wharton's jelly and derived cartilage cells. Cell Immunol 278: 35-44, 2012.

8. Huang P, Lin LM, Wu XY, et al: Differentiation of human umbilical cord Wharton's jelly-derived mesenchymal stem cells into germ-like cells in vitro. J Cell Biochem 109: 747-754, 2010.

9. Yang F, Yang D, Tu J, Zheng Q, Cai L and Wang L: Strontium enhances osteogenic differentiation of mesenchymal stem cells and in vivo bone formation by activating $\mathrm{Wnt} /$ catenin signaling. Stem Cells 29: 981-991, 2011.

10. Chao KC, Yang HT and Chen MW: Human umbilical cord mesenchymal stem cells suppress breast cancer tumourigenesis through direct cell-cell contact and internalization. J Cell Mol Med 16: 1803-1815, 2012.

11. Wang H, Qiu X, Ni P, et al: Immunological characteristics of human umbilical cord mesenchymal stem cells and the therapeutic effects of their transplantion on hyperglycemia in diabetic rats. Int J Mol Med 33: 263-270, 2014.

12. Le Blanc K: Immunomodulatory effects of fetal and adult mesenchymal stem cells. Cytotherapy 5: 485-489, 2003.

13. Nauta AJ and Fibbe WE: Immunomodulatory properties of mesenchymal stromal cells. Blood 110: 3499-3506, 2007.

14. Uccelli A, Moretta L and Pistoia V: Mesenchymal stem cells in health and disease. Nat Rev Immunol 8: 726-736, 2008.

15. Kon E, Muraglia A, Corsi A, et al: Autologous bone marrow stromal cells loaded onto porous hydroxyapatite ceramic accelerate bone repair in critical-size defects of sheep long bones. J Biomed Mater Res 49: 328-337, 2000.

16. Solchaga LA, Temenoff JS, Gao J, Mikos AG, Caplan AI and Goldberg VM: Repair of osteochondral defects with hyaluronanand polyester-based scaffolds. Osteoarthritis Cartilage 13: 297-309, 2005.

17. Amado LC, Saliaris AP, Schuleri KH, et al: Cardiac repair with intramyocardial injection of allogeneic mesenchymal stem cells after myocardial infarction. Proc Natl Acad Sci USA 102: 11474-11479, 2005.

18. Lin YT, Chern Y, Shen CK, et al: Human mesenchymal stem cells prolong survival and ameliorate motor deficit through trophic support in Huntington's disease mouse models. PloS One 6: e22924, 2011.

19. Miyahara Y, Nagaya N, Kataoka M, et al: Monolayered mesenchymal stem cells repair scarred myocardium after myocardial infarction. Nat Med 12: 459-465, 2006.

20. Di Nicola M, Carlo-Stella C, Magni M, et al: Human bone marrow stromal cells suppress T-lymphocyte proliferation induced by cellular or nonspecific mitogenic stimuli. Blood 99: 3838-3843, 2002.

21. Le Blanc K, Frassoni F, Ball L, et al: Mesenchymal stem cells for treatment of steroid-resistant, severe, acute graft-versus-host disease: a phase II study. Lancet 371: 1579-1586, 2008.

22. Le Blanc K, Rasmusson I, Sundberg B, et al: Treatment of severe acute graft-versus-host disease with third party haploidentical mesenchymal stem cells. Lancet 363: 1439-1441, 2004.

23. Mundra V, Gerling IC and Mahato RI: Mesenchymal stem cell-based therapy. Mol Pharm 10: 77-89, 2013.

24. Friedenstein AJ, Gorskaja JF and Kulagina NN: Fibroblast precursors in normal and irradiated mouse hematopoietic organs. Exp Hematol 4: 267-274, 1976.

25. Mennan C, Wright K, Bhattacharjee A, Balain B, Richardson J and Roberts S: Isolation and characterisation of mesenchymal stem cells from different regions of the human umbilical cord. Biomed Res Int 2013: 916136, 2013.

26. Roobrouck VD, Ulloa-Montoya F and Verfaillie CM: Self-renewal and differentiation capacity of young and aged stem cells. Exp Cell Res 314: 1937-1944, 2008.

27. Sarugaser R, Lickorish D, Baksh D, Hosseini MM and Davies JE: Human umbilical cord perivascular (HUCPV) cells: a source of mesenchymal progenitors. Stem Cells 23: 220-229, 2005.

28. Schellenberg A, Lin Q, Schuler H, et al: Replicative senescence of mesenchymal stem cells causes DNA-methylation changes which correlate with repressive histone marks. Aging 3: 873-888, 2011. 
29. Deuse T, Stubbendorff M, Tang-Quan K, et al: Immunogenicity and immunomodulatory properties of umbilical cord lining mesenchymal stem cells. Cell Transplant 20: 655-667, 2011.

30. Baksh D, Yao R and Tuan RS: Comparison of proliferative and multilineage differentiation potential of human mesenchymal stem cells derived from umbilical cord and bone marrow. Stem Cells 25: 1384-1392, 2007.

31. Weiss ML, Medicetty S, Bledsoe AR, et al: Human umbilical cord matrix stem cells: preliminary characterization and effect of transplantation in a rodent model of Parkinson's disease. Stem Cells 24: 781-792, 2006.

32. Fu YS, Cheng YC, Lin MY, et al: Conversion of human umbilical cord mesenchymal stem cells in Wharton's jelly to dopaminergic neurons in vitro: potential therapeutic application for Parkinsonism. Stem Cells 24: 115-124, 2006.
33. Lund RD, Wang S, Lu B, et al: Cells isolated from umbilical cord tissue rescue photoreceptors and visual functions in a rodent model of retinal disease. Stem Cells 25: 602-611, 2007.

34. Rachakatla RS, Marini F, Weiss ML, Tamura M and Troyer D: Development of human umbilical cord matrix stem cell-based gene therapy for experimental lung tumors. Cancer Gene Ther 14: 828-835, 2007.

35. Cho PS, Messina DJ, Hirsh EL, et al: Immunogenicity of umbilical cord tissue derived cells. Blood 111: 430-438, 2008.

36. Wang S, Yu L, Sun M, et al: The therapeutic potential of umbilical cord mesenchymal stem cells in mice premature ovarian failure. Biomed Res Int 2013: 690491, 2013.

37. Karahuseyinoglu S, Cinar O, Kilic E, et al: Biology of stem cells in human umbilical cord stroma: in situ and in vitro surveys. Stem Cells 25: 319-331, 2007. 